\title{
Impacts of tidal currents and Kuroshio intrusion on the generation of nonlinear internal waves in Luzon Strait
}

\author{
Tao Du, ${ }^{1,3}$ Yu-Heng Tseng, ${ }^{2}$ and Xiao-Hai Yan ${ }^{3,4}$ \\ Received 17 April 2007; revised 14 March 2008; accepted 1 April 2008; published 8 August 2008.
}

[1] The analysis of remotely sensed images shows that two kinds of nonlinear internal solitary waves (ISWs) in the forms of single ISW and groups of ISWs are commonly seen in Luzon Strait (LS), with larger occurrence during spring tides. A 2-D numerical model with idealized topography is then used to investigate the generation processes of ISWs in LS. The model results confirm that the ISWs are likely generated during spring tides with strong tidal currents. The results further suggest that the ISWs can be generated by two mechanisms: the evolution of depression and the internal mixing disturbance. Our simulations also show that the Kuroshio intrusion to LS or South China Sea through LS increases the occurrence of generating internal mixing and groups of ISWs. Both remotely sensed images and numerical simulations suggest the existence of a distance between the actual locations where ISWs are generated and where the strongest flow occurs.

Citation: Du, T., Y.-H. Tseng, and X.-H. Yan (2008), Impacts of tidal currents and Kuroshio intrusion on the generation of nonlinear internal waves in Luzon Strait, J. Geophys. Res., 113, C08015, doi:10.1029/2007JC004294.

\section{Introduction}

[2] Luzon Strait (LS), extending from the southern tip of Taiwan to Luzon of the Philippines in a north-south direction and connecting the Pacific Ocean and the SCS from east to west, has a very complicated bathymetry (see Figure 1). The Taiwan-Luzon (T-L) trench, which is very deep, is located in the middle of the strait. On the eastern side of the trench, a shallow ridge runs along $121.5^{\circ} \mathrm{E}$, on which the Batan Islands, Babuyan Islands, and many undersea sills are located. Some shallow and narrow channels (bold lines labeled 1 through 8 in Figure 1) are located in the Batan and Babuyan Islands, with the most shallow and narrow channel (labeled 5) having minimum depth and width of approximately $155 \mathrm{~m}$ and $3.5 \mathrm{~km}$, respectively. On the western side of the trench, only undersea sills exist.

[3] LS is believed to be an important source area of the nonlinear internal waves observed in the northern part of the SCS, for which many hypotheses and theories on the generation of the internal waves in LS have been suggested [Liu et al., 1998; Du, 2000; Hsu et al., 2000; Orr and Mignerey, 2003; Zhao et al., 2004; Ramp et al., 2004; Liu et al., 2004; Duda et al., 2004; Lien et al., 2005; Yuan et al., 2006]. Ebbesmeyer et al. [1991] and Bole et al. [1994]

\footnotetext{
${ }^{1}$ Physical Oceanography Laboratory, Ocean University of China, Qingdao, China.

${ }^{2}$ Department of Atmospheric Sciences, National Taiwan University, Taipei, Taiwan.

${ }^{3}$ Center for Remote Sensing, Graduate College of Marine Studies, University of Delaware, Newark, Delaware, USA.

${ }^{4}$ State Key Laboratory of Marine Environmental Science, Xiamen University, Xiamen, China.
}

Copyright 2008 by the American Geophysical Union. 0148-0227/08/2007JC004294\$09.00 believed that the interaction of tides with the sill in a particular channel of LS (labeled 5 in Figure 1) causes the generation of a sequence of ISWs propagating toward the SCS. Liu et al. proposed that nonlinear internal waves may be generated by the Kuroshio intrusion into the SCS via LS. Hsu et al. suggested that ISWs observed in the SCS are of tidal origin, generated through a lee-wave mechanism in the strait. Zhao et al. categorized ISWs observed in the northern part of the SCS into two types: single ISW and groups of ISWs. They believed that the birthplace of ISWs is the shallow ridge running along $121.5^{\circ} \mathrm{E}$ in LS. They showed that a single ISW is generated by nonlinear steepening of baroclinic tides or large depressions, rather than a lee-wave mechanism. On the other hand, groups of ISWs might result from a lee-wave mechanism or the evolution of baroclinic tides; however, no further evidence is given. Ramp et al. proposed that the most possible source for ISWs observed in the northern part of the SCS was over the ridge near the Batan Islands. Lien et al. investigated the energy budget of nonlinear internal waves in the SCS; they also discussed some possible mechanisms for nonlinear internal waves in LS, such as lee-wave mechanism and nonlinear internal tide mechanism. They believed that the observed nonlinear internal waves in the northern part of the SCS are generated by nonlinear internal tides in LS, rather than lee waves. More recently, Yuan et al. suggested that the instability of the Kuroshio west wing in LS serves as a disturbance source for internal waves in LS.

[4] Though none of the hypotheses and theories on the generation of nonlinear internal waves in LS has been verified by in-situ observations, tidal currents and the Kuroshio intrusion to the SCS are thought to play an important role. The motivation of the present work is to investigate the impacts of tidal currents and the Kuroshio 


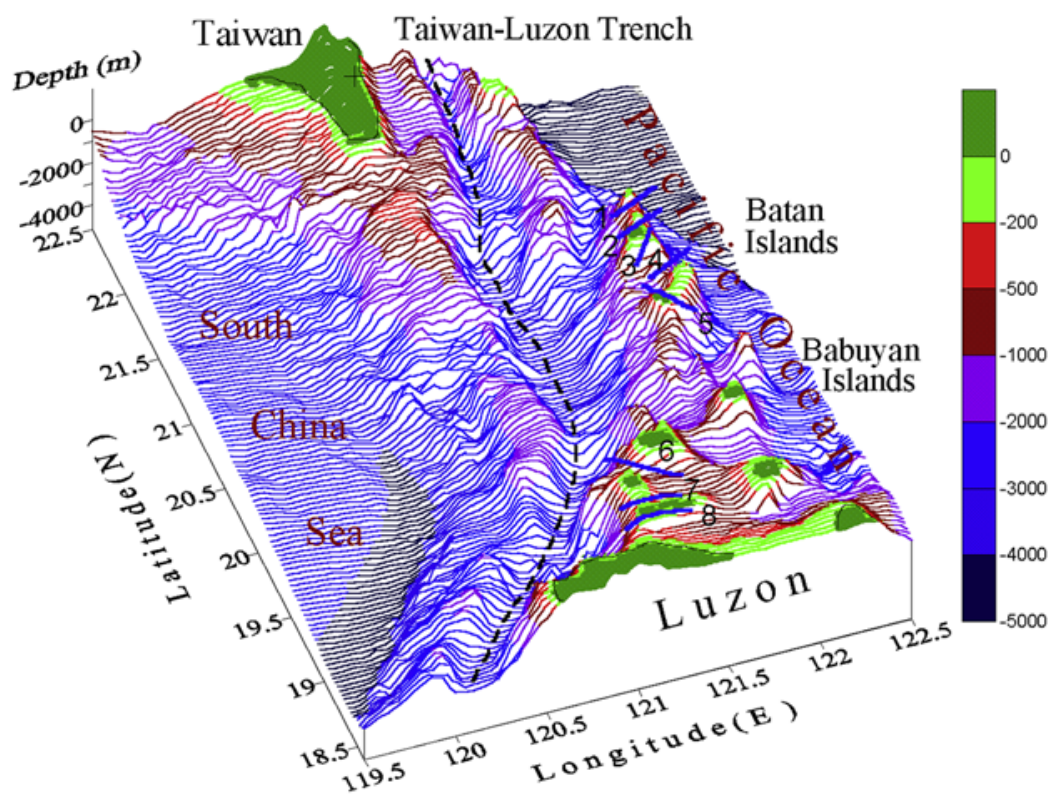

Figure 1. The topography in Luzon Strait (data from http://topex.ucsd.edu/cgi-bin/get_data.cgi).

intrusion to the SCS on the generation of internal waves in LS. This is done by analyzing remotely sensed images in combination with simplified 2-D numerical simulations. The remotely sensed images which show detailed sea surface signature of ISWs in LS are analyzed in section 2. A 2-D numerical model is further used to verify the generation processes of ISWs under various combinations of tidal currents and the Kuroshio intrusion in section 3 . Some discussions on the numerical results and possible generation mechanisms for ISWs in LS are presented in section 4. Finally, conclusions are given in section 5 .

\section{Remotely Sensed Satellite Images}

[5] Sea surface signatures of nonlinear internal waves in the northern part of the SCS have been frequently observed from the remotely sensed images, as documented in many studies [e.g., Liu et al., 1998; Zhao et al., 2004; Fang and $D u, 2005]$. Some nonlinear internal waves appear as groups of ISWs (Figure 2), while others as a single ISW (Figure 3). The ISWs in Figure 2 (images taken on 16 June 1995 and superimposed on the bathymetry of LS) can be divided into two parts. The northern part (part 1 in Figure 2) mainly propagates westward, while the southern part (part 2 in Figure 2) propagates northwestward. The two different propagating directions indicate that the northern and southern parts of the ISWs probably originate from different source locations. The northern ISWs may result from the interactions of currents with the Batan Islands topography, while the southern ones may be induced by the interactions in the Babuyan Islands. Similarly, the ISWs in Figure 3 can also be divided into two parts, each originating from a different source area. The images in Figures 2 and 3 clearly illustrate that both types of ISWs can have very large crests that spread across the entire strait. In contrast, the images in Figure 4 show that ISWs can also have shorter crests. These short crest ISWs were observed in a location near $121^{\circ} \mathrm{E}$, much closer to the Batan Islands (Figure 4).

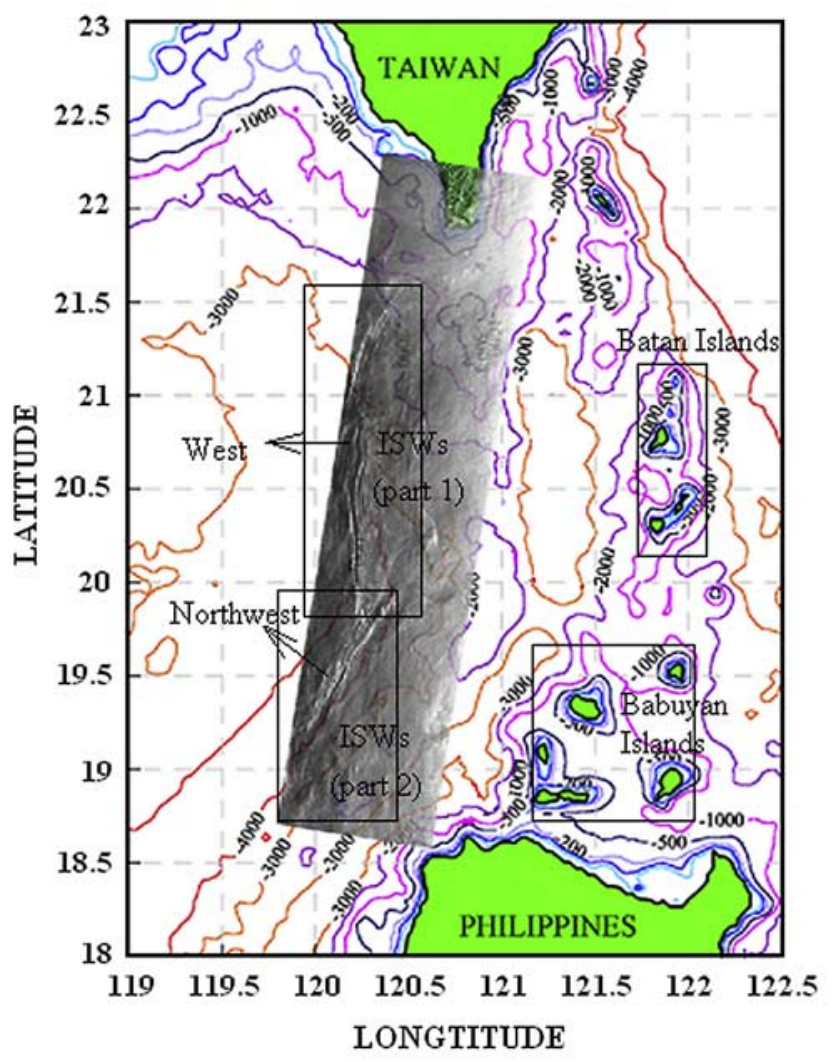

Figure 2. An ERS-1 SAR image $(100 \mathrm{~km} \times 500 \mathrm{~km}$, taken on 16 June 1995) composition, which consists of five standard images $(100 \mathrm{~km} \times 100 \mathrm{~km})$, showing sea surface radar signatures of the ISWs propagating westward toward the South China Sea, superimposed on the bathymetry of Luzon Strait. The leading soliton has a crest length exceeding $200 \mathrm{~km}$ 


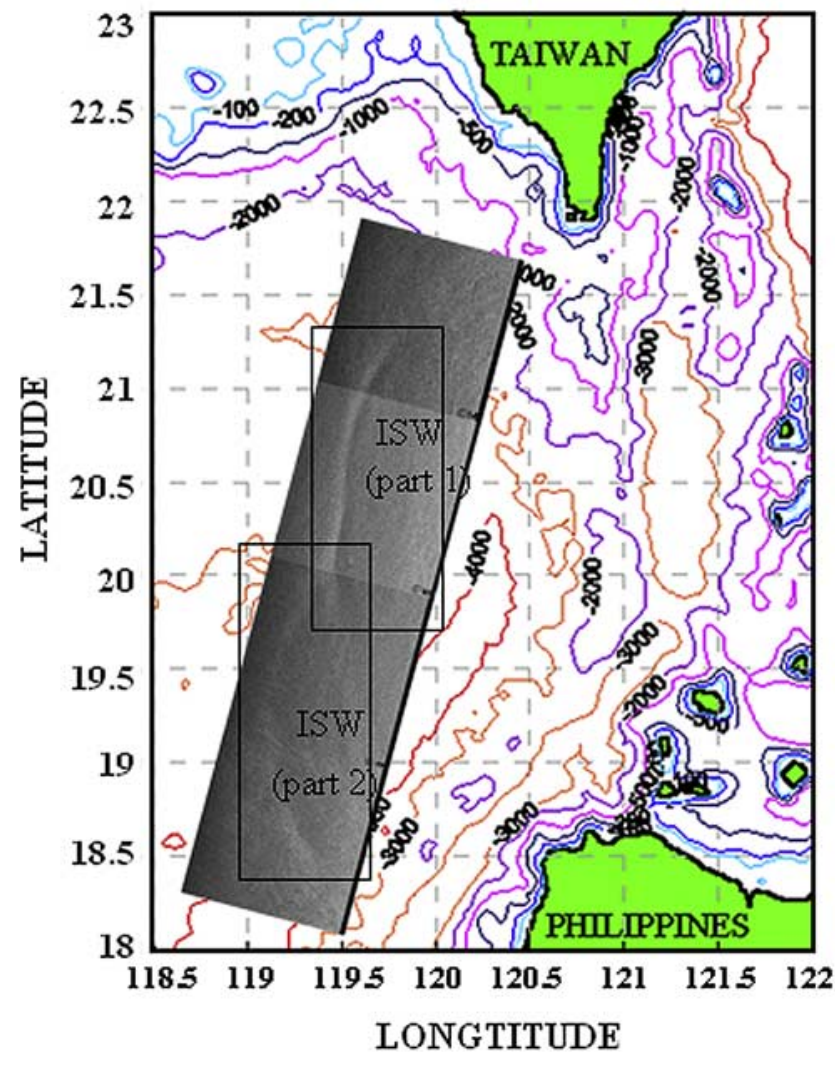

Figure 3. An ERS-1 SAR composite image $(100 \mathrm{~km} \times$ $400 \mathrm{~km}$, taken on 9 August 1995), which consists of four standard images $(100 \mathrm{~km} \times 100 \mathrm{~km})$, showing sea surface radar signatures of the single ISW propagating westward toward the SCS from LS. The bathymetry is superimposed in meters.

[6] We analyzed a total of 711 standard images taken from European Remote Sensing satellite 1/2 (ERS 1/2), Satellite Pour l'Observation de la Terre (SPOT) 1 to 5, Radasat, Envisat, and Landsat. These standard images constitute 211 composite images, representing observation events taken unevenly from 1995 to 2005. For example, the composite image in Figure 2 is composed of five standard images, four of which clearly show the signature of ISWs. Eighty-five composite images captured the sea surface signature of ISWs near LS in our analysis. Figure 5 shows the spatial distribution of all observed ISWs (black lines represent ISW crests). It is noteworthy that all ISWs appear in the area west of $121^{\circ} \mathrm{E}$. In particular, the ISWs close to $121^{\circ} \mathrm{E}$ usually have short crests. The shortest distance between the Batan Islands and the observed ISWs is about $100 \mathrm{~km}$ (Figure 5, S-Batan), while the shortest distance between the Babuyan Islands and the observed ISWs is about $40 \mathrm{~km}$ (Figure 5, S-Babuyan).

[7] A few ISWs are also observed north of Luzon; however, none has been observed in the area over the shallow ridge along $121.5^{\circ} \mathrm{E}$ (Figure 1). Although the images in Figures 2-4 show the ISWs that occurred only during the summer, many other images illustrate that ISWs can occur in LS all year around.

[8] To better illustrate the relationship between the occurrence frequency of ISWs and the strength of tidal currents in LS, we compare the number of observation events in which ISWs are observed (yellow bars) and the total observation events on each lunar day (blue bars) in Figure 6 . The lunar month is the time between two successive similar syzygies, and is measured from New Moon to New Moon. Interestingly, most ISWs are observed during spring tides, i.e., in the new moon (lunar day 29, 30, 1-4) and full moon (lunar day 14-19) periods (Figure 6). The occurrence frequency of ISWs on each lunar day shows a similar trend, although there is a lag of a few days between the day with maximum frequency and the new (or full) moon days (Figure 7). This indicates that ISWs are more likely generated during spring tides than neap tides. Since tidal currents are stronger during spring tides than neap tides, the generation of ISWs in LS should be highly correlated to the strength of tidal currents; the stronger the tidal currents, the more likely ISWs are generated. The higher occurrence rate of ISWs during spring tides in LS has also been indicated in previous studies based on in-situ observations in the SCS [e.g., Ramp et al., 2004; Duda et al., 2004; Fang and Du, 2005].

[9] The occurrence frequency of ISW is also higher on the 21st and 22nd days of a lunar month (Figure 7), just prior to neap tides. Since tidal currents are weak on these days, this indicates that tidal currents may not be the only contributor to the generation of ISWs in LS.

[10] To further examine the relationship, we compared ISW observations, the strength of tidal currents, and the Kuroshio intrusion to SCS on two proximate days, 19 and 22 July 2001. The ISW shown in an ERS-2 SAR image taken on 19 July 2001 (Figure 8b) is a single westward one. According to the composite image in Figure 3, we may suggest that a single ISW occurred over the entire LS on that day. ISWs observed in a SPOT-2 image taken on 22 July 2001 are groups of westward ISWs. We may also suggest that the groups of ISWs occurred over the entire LS, similar as shown in Figure 2. On these two days, the maximum tidal current should be strong because both days are within a single spring tide. Since 19 July is two days prior to a new noon while 22 July is one day following the new moon, the maximum tidal current on 22 July should be stronger than that on 19 July. Besides tidal currents, the Kuroshio intrusion to the SCS also flows though LS during this period. Figure 8a shows the assimilated local circulation in LS at a depth of $100 \mathrm{~m}$ (white arrows) on 19 July 2001 using the Naval Research Laboratory (NRL) North Pacific Nowcast/ Forecast system. Figure 9a presents the local circulation for the same region on 22 July 2001. The simulated circulation shows that the Kuroshio intrusion to the SCS is weak on both days, with a westward current of approximately $20 \mathrm{~cm} / \mathrm{s}$ in the islands. This intrusion current is weak compared with both the observed tidal current [Ebbesmeyer et al., 1991] and the intrusion current at other times [Centurioni et al., 2004]. The simulation also showed that there are no significant changes in the circulation pattern passing through the islands on both days (Figures 8a and 9a). This implies that on these two days, strong tidal currents play a more important role in the generation of the ISWs than the weak Kuroshio intrusion. It also implies that stronger tidal currents may cause the generation of groups of ISWs.

[11] To summarize, our analysis of the remotely sensed images strongly suggest the following key issues. 


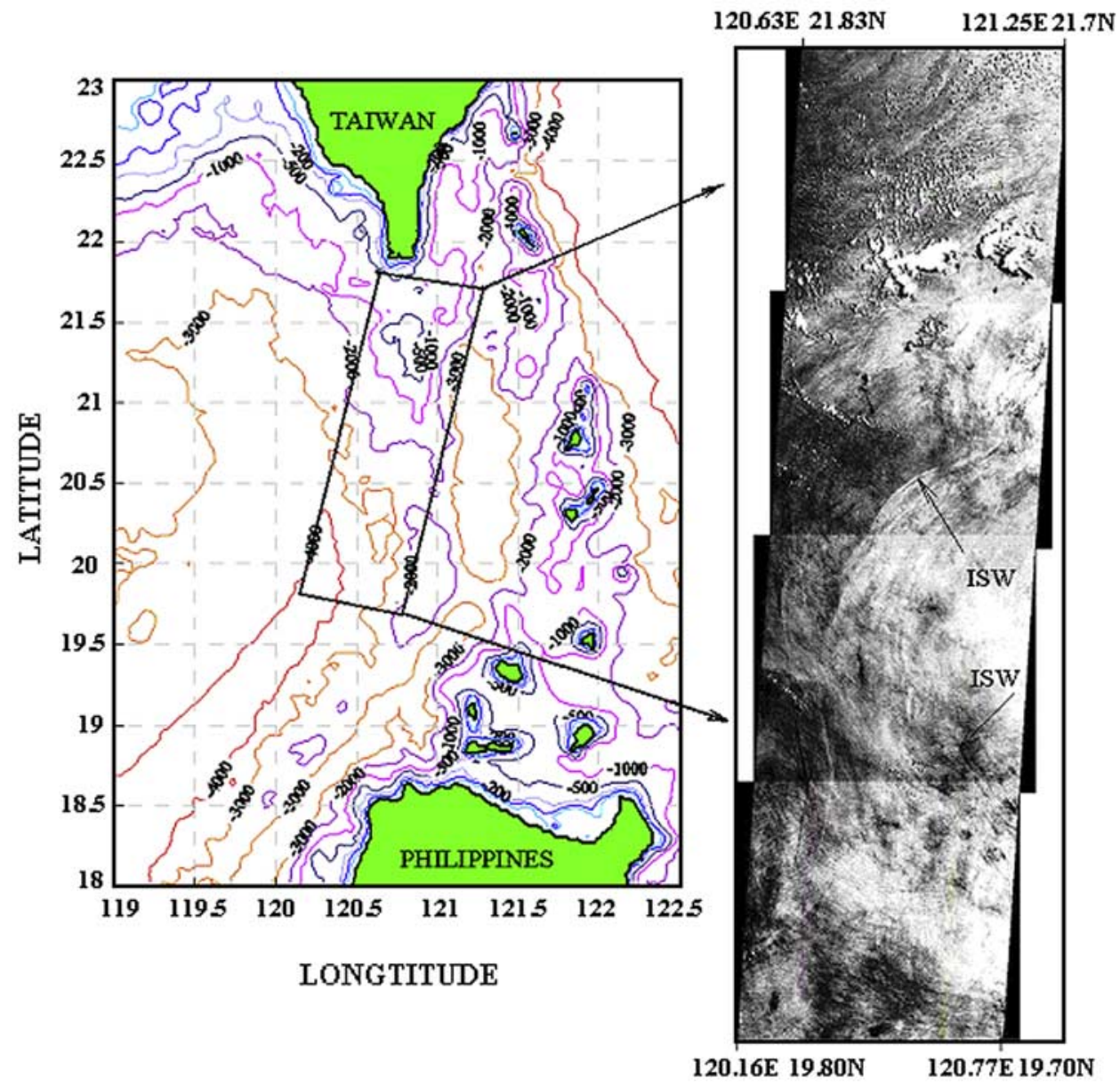

Figure 4. A composite of four images from Satellite Pour l'Observation de la Terre (SPOT) 2 (taken on 4 July 2003), showing the internal solitary waves occurring near $121^{\circ} \mathrm{E}$.

[12] 1. There are at least two kinds of nonlinear internal waves generated in LS: single ISW and groups of ISWs.

[13] 2. The generation of ISWs in LS closely relates to the strength of tidal currents. ISWs are more likely generated during spring tides when tidal currents are strong.

[14] 3. ISWs can be generated in LS in all months of a year.

[15] 4. ISWs can be generated from two possible sources that may be related to the Batan and Babuyan Islands, respectively.

[16] 5. Tidal currents may not be the only contributors that impact the generation of ISWs in LS.

\section{Numerical Simulation}

\subsection{The Background of Currents in LS}

[17] LS is a major passage for astronomical tides propagating directly from the Pacific Ocean into the SCS [Fang et al., 1999; Niwa and Hibiya, 2004]. Therefore periodic tidal currents play an important role in the strait. Based on numerical studies [Zhao and Alford, 2006; Fang et al., 1999], tidal currents are weak in most parts of the strait and on an east-west direction. However, tidal currents can become very strong when flowing over the shallow ridge or through the narrow and shallow channels in the islands
(Figure 1). For example, the observed speeds of tidal currents could reach $283 \mathrm{~cm} / \mathrm{s}$ in a shallow and narrow channel (Figure 1, channel 5) of the Batan Islands [Ebbesmeyer et al., 1991].

[18] Several studies show that, in addition to tidal currents, zonal currents also exist in LS; the intrusion of the Kuroshio to the SCS via LS is the most important one among them [Liu et al., 2000; Chu and Li, 2000; Li et al., 2002]. Observations show that a portion of the Kuroshio water frequently passes through LS and enters the SCS between October and March; during other periods, the Kuroshio water enters LS only from its southeastern and central parts (flowing through at least the Batan Islands) and exits toward its northeastern part [Centurioni et al., 2004]. Hereafter, we refer to the intrusion of the Kuroshio water to the SCS (or only into LS) as the Kuroshio branch (K-branch). The K-branch has a near-surface daily averaged velocity of $70 \pm 40 \mathrm{~cm} / \mathrm{s}$ when it crosses the $120.8^{\circ} \mathrm{E}$ line as it enters the SCS; a maximum near-surface daily averaged velocity of $165 \pm 1 \mathrm{~cm} / \mathrm{s}$, almost westward, was observed in December 1997 [Centurioni et al., 2004].

[19] The resulting flow from tidal currents and the K-branch can reach a very high speed when passing over the shallow ridge or the shallow and narrow channels, as tidal 


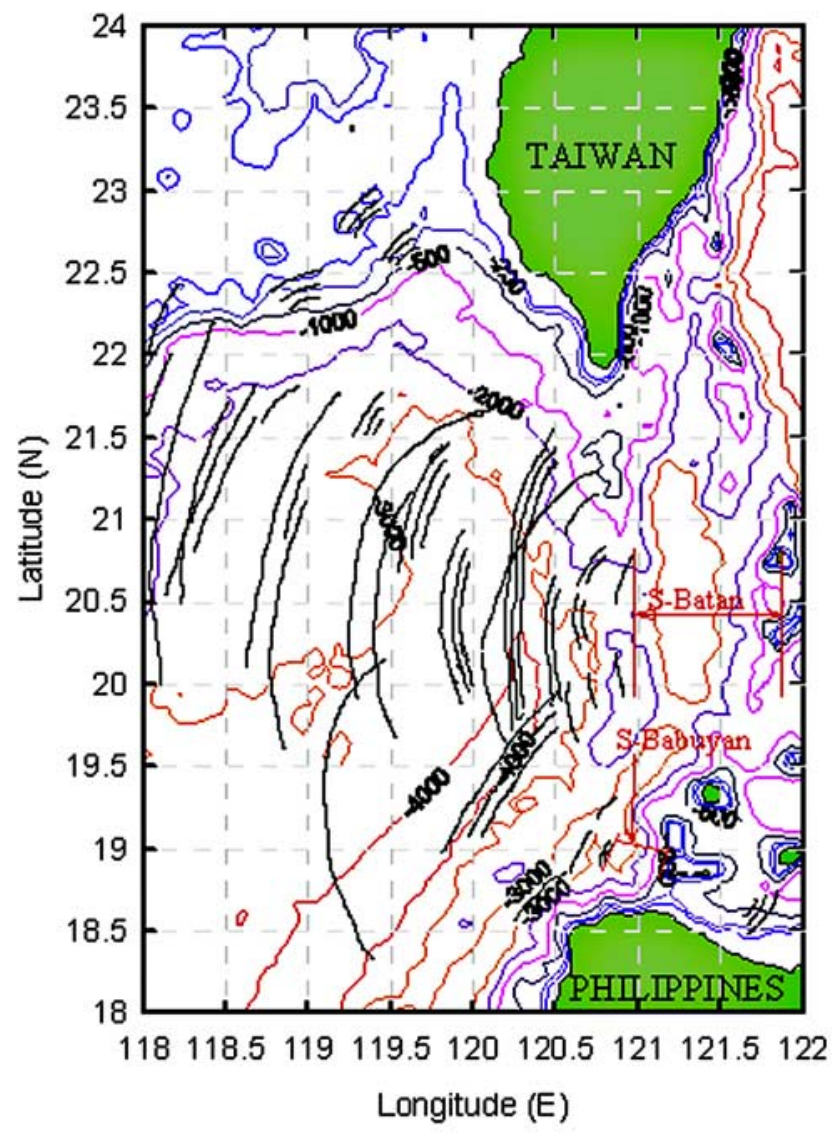

Figure 5. The spatial distribution of ISWs near LS based on the ISWs' sea surface signatures on remotely sensed images.

currents do in LS. Therefore both tidal currents and the K-branch should have important impacts on the generation of ISWs in LS.

\subsection{The Numerical Model and Its Setups}

[20] In order to further verify the findings analyzed from the remotely sensed images in section 2 and study the impacts of tidal currents and the Kuroshio intrusion on the generation of ISWs in LS, we used a simplified twodimensional numerical model to assess the generation process of ISWs. A fourth-order accurate, hydrostatic Dietrich/Ceter for Air-sea technology (DieCAST) model [Tseng and Dietrich, 2006; Dietrich, 1997] is used to quantify the influences of various flow conditions. The numerical model solves the primitive equation set. The high-order, nondissipative numerical scheme provides high computational accuracy and low numerical dissipation and dispersion, which is particularly useful for investigating nonlinear internal waves and their associated turbulent mixing.

[21] The simulation domain is shown in Figure 10a with two idealized seamounts. The higher one has a minimum depth $\sim 300 \mathrm{~m}$ and is $100 \mathrm{~km}$ away from the eastern open boundary, representing the Batan or the Babuyan Islands. The other has $1300 \mathrm{~m}$ depth on its top and locates $215 \mathrm{~km}$ away from the eastern boundary, representing the undersea sills west of the T-L trench. The domain size is $400 \mathrm{~km} \times$ $3000 \mathrm{~m}$. Only the upper $2500 \mathrm{~m}$ is shown in Figure 10 to highlight the upper layer stratification. A flat bottom of $3000 \mathrm{~m}$ depth is used. The horizontal resolution is $160 \mathrm{~m}$ while the vertical is geometrically stretched with $4 \mathrm{~m}$ at the top layer and 60 levels totally. The tidal and K-branch currents are forced at the eastern boundary. The domain is long enough to allow the westward waves to propagate downstream without any reflection during the simulation periods. Open boundary conditions are used at both sides while additional absorbing boundary condition is imposed near the eastern boundary with exponentially decayed buffer layer to avoid unrealistic/unwanted ISWs due to reflection. Figure 10b shows the surface longitudinal velocity across the LS within four consecutive years from a fully three dimensional, duo-grid North Pacific Ocean model (resolution is $1 / 8^{\circ}$ in the western Pacific). The observed ISWs (Figures 2 and 3) closely relate to the westward Kuroshio intrusion spatially, which is frequently seen in the south of LS. Some lee waves and ISWs originating above the topography could also propagate upstream (eastward). However, the main focus of this study is the ISWs propagating westward toward the SCS, these eastward propagating ISWs are beyond the scope of this study and absorbed by the buffer layer.

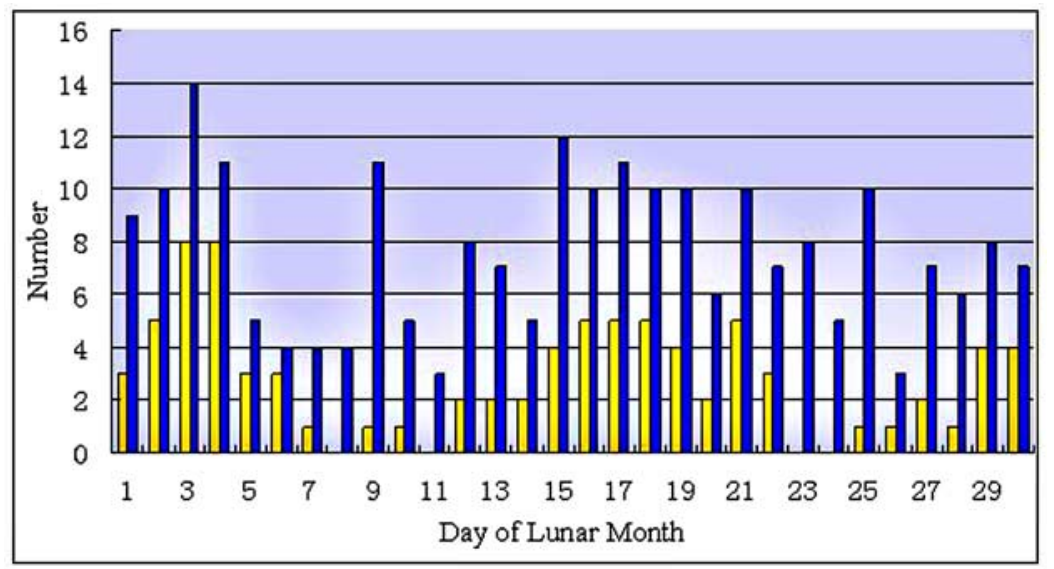

Figure 6. The total number of observation events (blue bars) and the number of events with the observed ISWs (yellow bars) on each day of a lunar month in LS. 


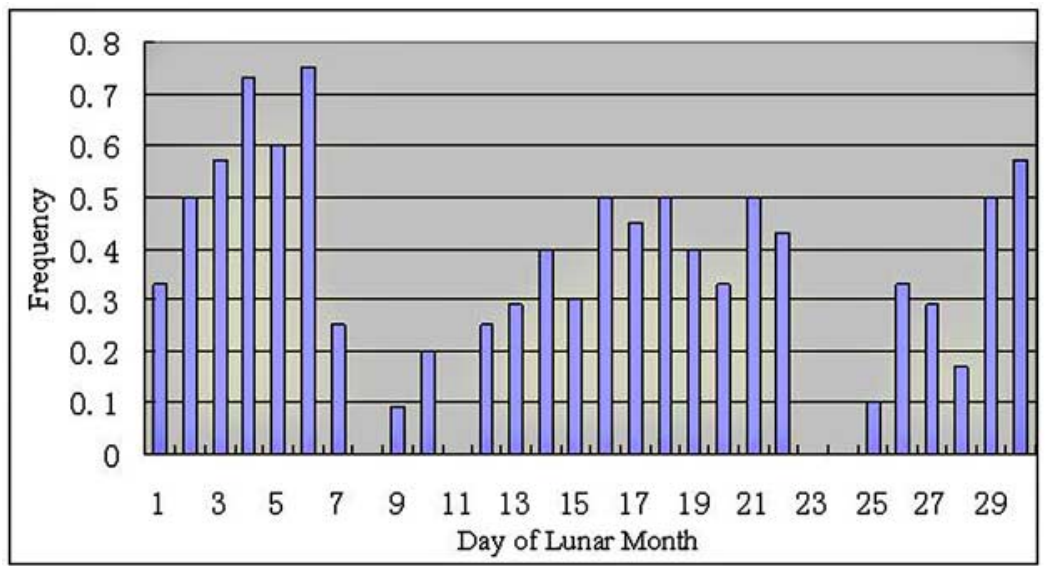

Figure 7. The occurrence frequency of ISWs on each day of a lunar month in LS.

[22] The time step is $2 \mathrm{~s}$. Vertical mixing is parameterized using a modified approach of Pacanowski and Philander [1981]. This widely used vertical mixing parameterization takes both the vertical shear and the vertical stratification into account. Note that this vertical parameterization is not accurate enough to resolve detailed overturning and mixing, such as the three dimensional vortex structures resulting from coastal upwelling [e.g., Tseng and Ferziger, 2001]. Further, weakly nonlinear internal waves are characterized by a delicate nonhydrostatic balance between nonlinear
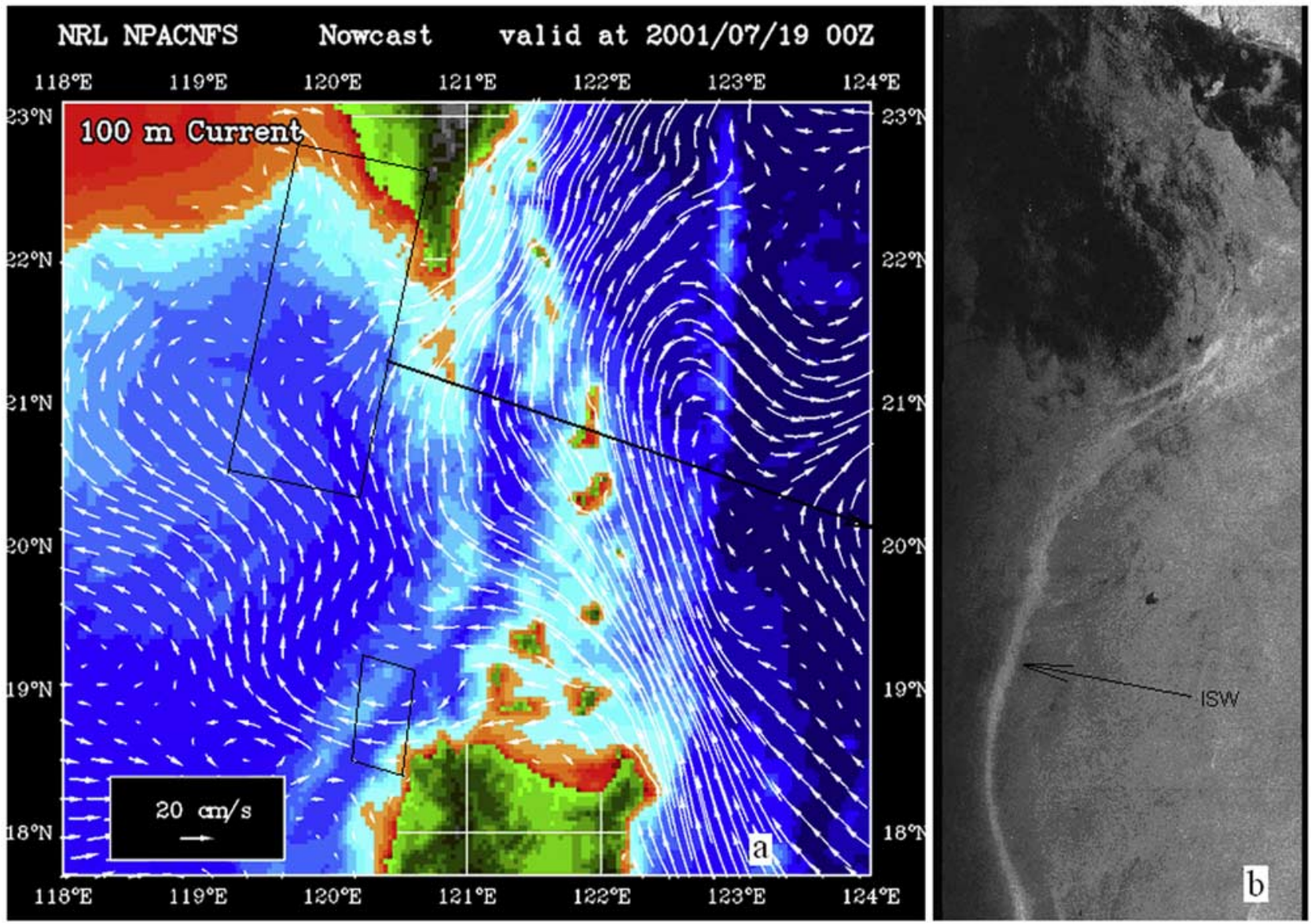

Figure 8. (a) The current vectors at $100 \mathrm{~m}$ depth (white arrows) on 19 July 2001, which are computed by the Naval Research Laboratory (NRL) North Pacific Nowcast/Forecast system, superimposed by the topography (colors) around LS. (b) An ERS-2 SAR image taken on 19 July 2001, which displays a single westward ISW generated in the strait. 


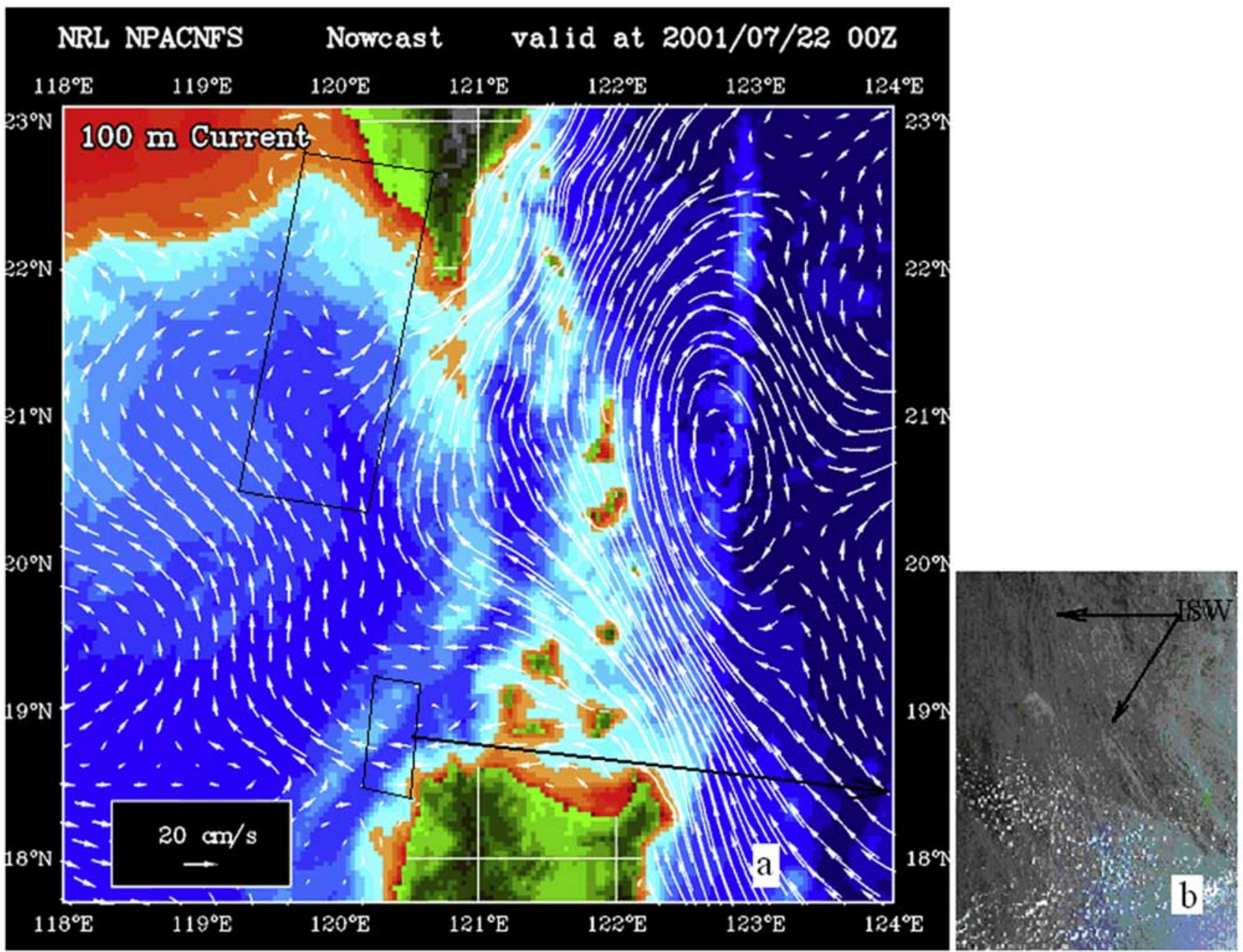

Figure 9. (a) The current vectors at $100 \mathrm{~m}$ depth (white arrows) on 22 July 2001, which are computed by the NRL North Pacific Nowcast/Forecast system, superimposed by the topography (colors) around LS. (b) An ERS-2 SAR image taken on 22 July 2001, which displays packet of westward ISWs generated in the strait.

steepening and dispersion [Venayagamoorthy and Fringer, 2007]. Nonhydrostatic dispersion may lead to the formation of wave packets with fewer peaks than the hydrostatic one so that a nonhydrostatic model is essentially required to simulate such detailed formation processes [Tseng et al., 2005; Tseng and Breaker, 2007]. Two-dimensionality also limits the growth of nonlinear instabilities. We emphasize that the detailed ISWs formation and the subsequent processes are lack in our idealized study and are only qualitatively simulated here. Nevertheless, the present idealized study is appropriate to quantify the influences of flow conditions in the two-dimensional idealized setting since the effects of these details are qualitatively parameterized. The present simulations also provide useful guidelines for further numerical studies of ISWs in this area. In order to clarify these nonhydrostatic influences, a fully threedimensional, high-resolution, nonhydrostatic numerical study is now carried out within a fully coupled North Pacific Ocean Model framework (Y. H. Tseng et al., Seasonal variability and the dynamics of Kuroshio intrusion into the Luzon and Taiwan Straits, manuscript in preparation, 2008).
Further investigation and results will be presented in a consequent paper and will not be duplicated herein.

[23] The initial temperature is also shown in Figure 10 with thermocline at $150 \mathrm{~m}$ depth. At the eastern open boundary of the domain, an inflow is imposed such that

$$
V=V_{K}+V_{t} \sin (2 \pi t / T)
$$

where $V_{K}$ represents a mean $\mathrm{K}$-branch current, $V_{t}$ is the amplitude of a tidal current, $T$ is the period of the tidal current and it equals to $12 \mathrm{hr}$ in the simulations, and $t$ is time. To study the influences of different flow conditions on the generation of ISWs, we have carried out eight different simulations. The detailed flow conditions and values are given in Table 1, where $V_{m}$ is the maximum velocity at the top of the seamount near the eastern boundary. In Case A, only a weak tide is imposed at the eastern open boundary; it brings up a maximum velocity $68 \mathrm{~cm} / \mathrm{s}$. In Case B, a strong $\mathrm{K}$-branch and a weak tide are imposed simultaneously, and $V_{m}$ is $252 \mathrm{~cm} / \mathrm{s}$, while in Case $\mathrm{B}^{\prime}$, a medium K-branch and the weak tide generate $V_{m}=183 \mathrm{~cm} / \mathrm{s}$. In Case C, only a 

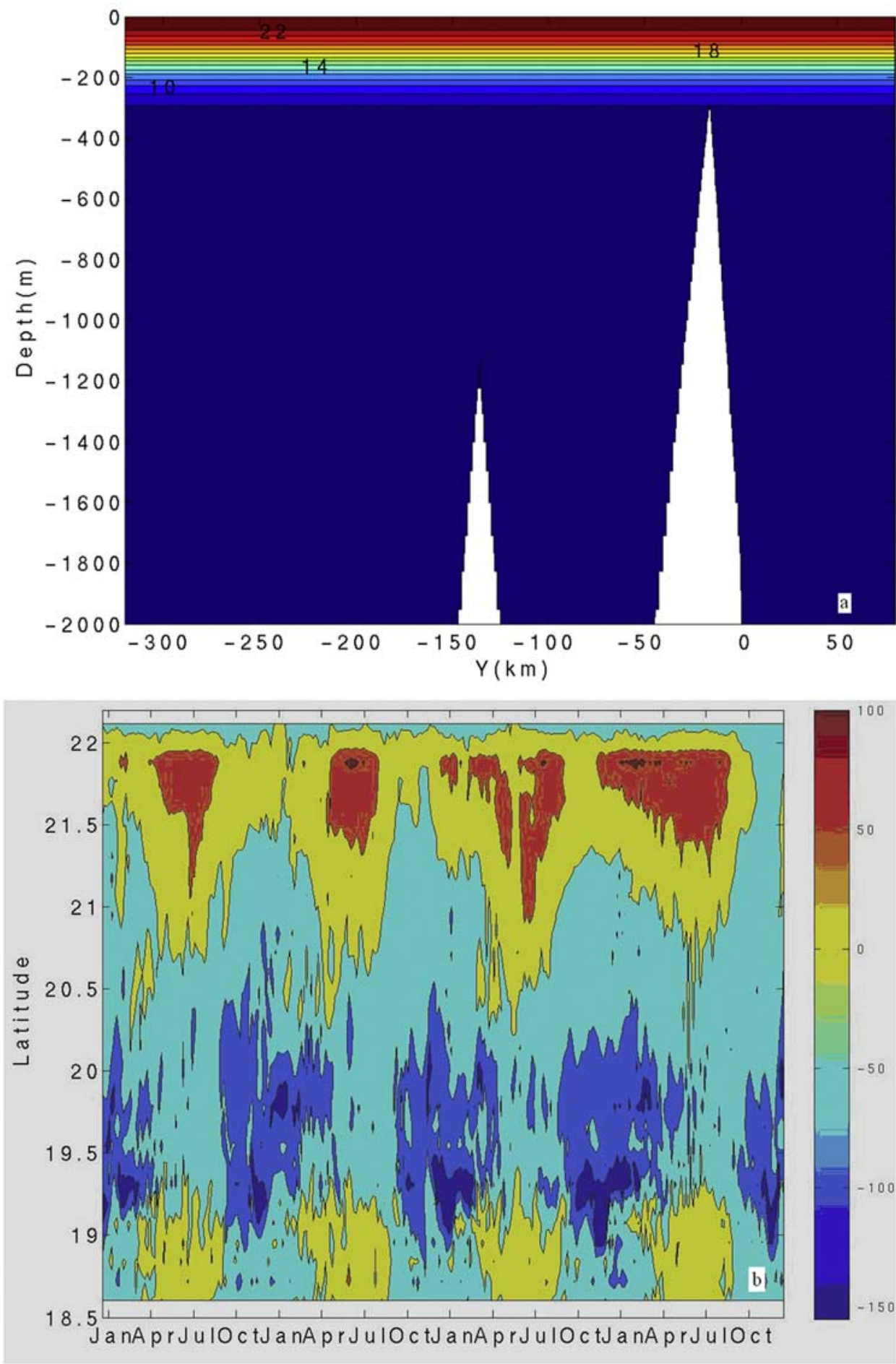

Figure 10. (a) The initial stratification and partial topography in the domain. The higher seamount (right seamount) has a minimum depth of $300 \mathrm{~m}$, and the lower seamount (left seamount) has $1300 \mathrm{~m}$. The thermocline locates at $\sim 150 \mathrm{~m}$ depth. (b) Surface longitudinal velocity across the LS within four consecutive years from a duo-grid North Pacific Ocean model (resolution is $1 / 8^{\circ}$ in the western Pacific).

medium tide is imposed, and $V_{m}$ reaches $146 \mathrm{~cm} / \mathrm{s}$. In Case D, a medium tide and a strong K-branch are imposed simultaneously, bringing a $V_{m}$ of $290 \mathrm{~cm} / \mathrm{s}$, while in Case $\mathrm{D}^{\prime}$, the medium tide and a weak K-branch make a $V_{m}$ of only $161 \mathrm{~cm} / \mathrm{s}$. In Case E, a single strong tide is imposed, giving a $V_{m}$ of $199 \mathrm{~cm} / \mathrm{s}$, while in Case $\mathrm{F}$, when a strong
K-branch is superimposed on the strong tide, $V_{m}$ becomes $326 \mathrm{~cm} / \mathrm{s}$, the maximum velocity in all simulations.

\subsection{Simulation Results}

[24] The numerical sensitivity tests provide significant insights on the different generation processes of ISWs under 
Table 1. Inflow Conditions Used in the Numerical Experiments

\begin{tabular}{|c|c|c|c|c|c|c|c|c|}
\hline \multirow[b]{2}{*}{ Cases, $\mathrm{cm} / \mathrm{s}$} & \multicolumn{3}{|c|}{ Weak Tide } & \multicolumn{3}{|c|}{ Medium Tide } & \multicolumn{2}{|c|}{ Strong Tide } \\
\hline & $\begin{array}{c}\text { Case } \\
\text { A } \\
\end{array}$ & $\begin{array}{c}\text { Case } \\
\text { B } \\
\end{array}$ & $\begin{array}{c}\text { Case } \\
\text { B }^{\prime} \\
\end{array}$ & $\begin{array}{c}\text { Case } \\
\mathrm{C} \\
\end{array}$ & $\begin{array}{c}\text { Case } \\
\text { D } \\
\end{array}$ & $\begin{array}{c}\text { Case } \\
\mathrm{D}^{\prime} \\
\end{array}$ & $\begin{array}{c}\text { Case } \\
\text { E }\end{array}$ & $\begin{array}{c}\text { Case } \\
\mathrm{F} \\
\end{array}$ \\
\hline$V_{K}$ & 0 & 20 & 10 & 0 & 20 & 4 & 0 & 20 \\
\hline$V_{t}$ & 10 & 10 & 10 & 20 & 20 & 20 & 30 & 30 \\
\hline$V_{m}$ & 68 & 252 & 183 & 146 & 290 & 161 & 199 & 326 \\
\hline
\end{tabular}

different flow conditions. The time-series temperature fields taken from the second period are shown in Figures 11-13. Figure 11 illustrates different generation processes of ISWs in one period when only a weak (left, Case A), medium (middle, Case C), or strong tide (right, Case E) is imposed at the eastern open boundary, respectively. Each process is composed of five sequential phases within one period. Only the upper $400 \mathrm{~m}$ region between the two seamounts is shown every 144 minutes. When a weak tide is imposed (Case A, left), a depression occurs west of the higher seamount at approximately 0.6 day. As the depression continues to move westward, no ISWs are generated from the depression. When a medium tide is imposed (Case C, middle), the depression becomes steeper (0.6 day). ISWs are generated from the development of the depression ( 0.7 day, $\sim 20 \mathrm{~km}$ away from the top of the higher seamount) and then propagate away westward. When a strong tide is imposed (Case E, right), the initial depression becomes much steeper and a group of ISWs are generated directly from the steeping depression (0.70 day, $15 \mathrm{~km}$ away from the top of the higher seamount). A small-scale single depression (about $6 \sim 8 \mathrm{~km}$ wide) also occurs on 0.8 day at a position about $50 \mathrm{~km}$ away from the top of the higher seamount. This small-scale single depression resembles the
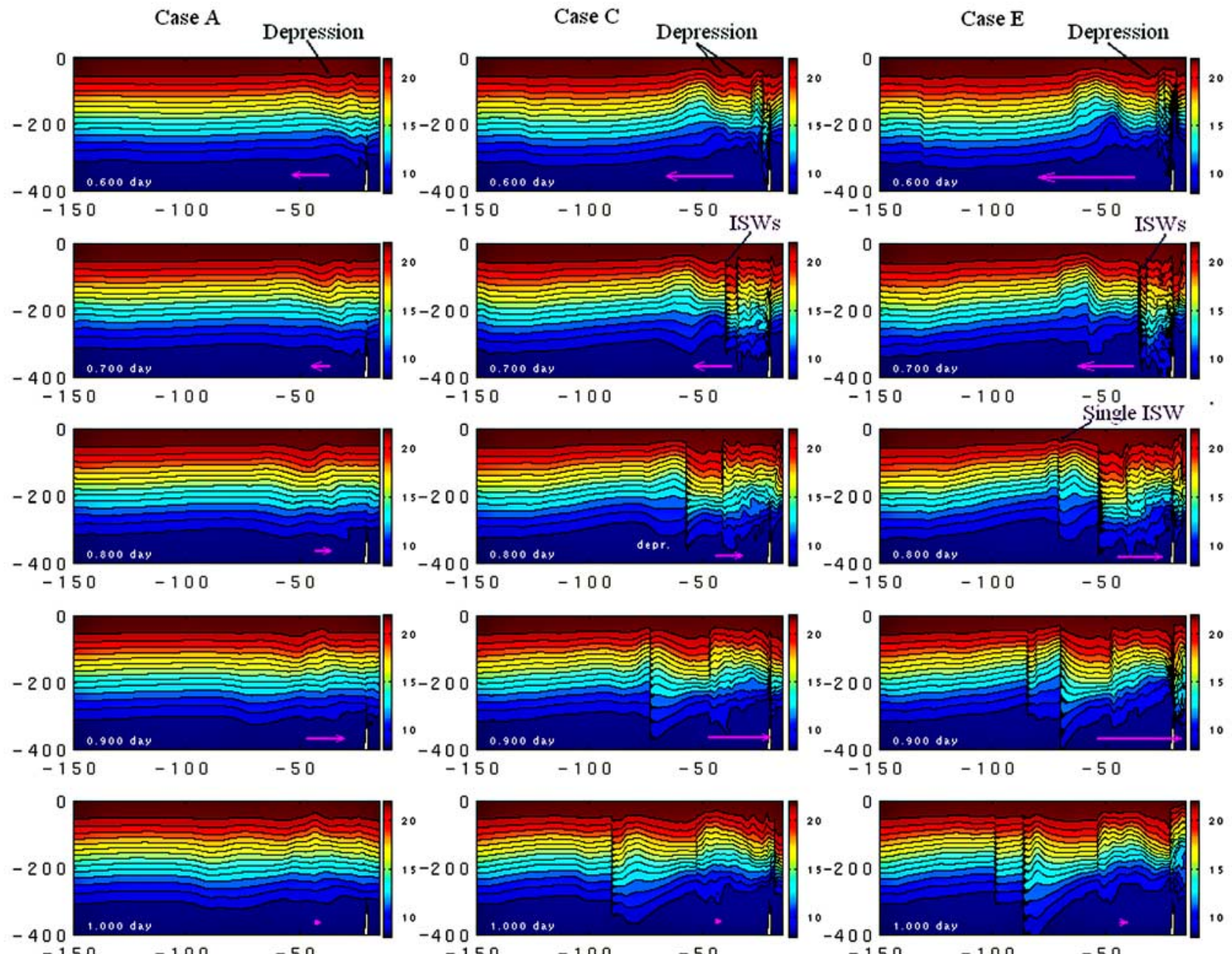

Figure 11. The temperature evolution showing the generation of ISWs within one tidal cycle (only a part of the simulation domain is shown) in different flow conditions without the K-branch: weak (left), medium (middle), and strong tide (right), respectively. The contour interval is $1^{\circ} \mathrm{C}$. 

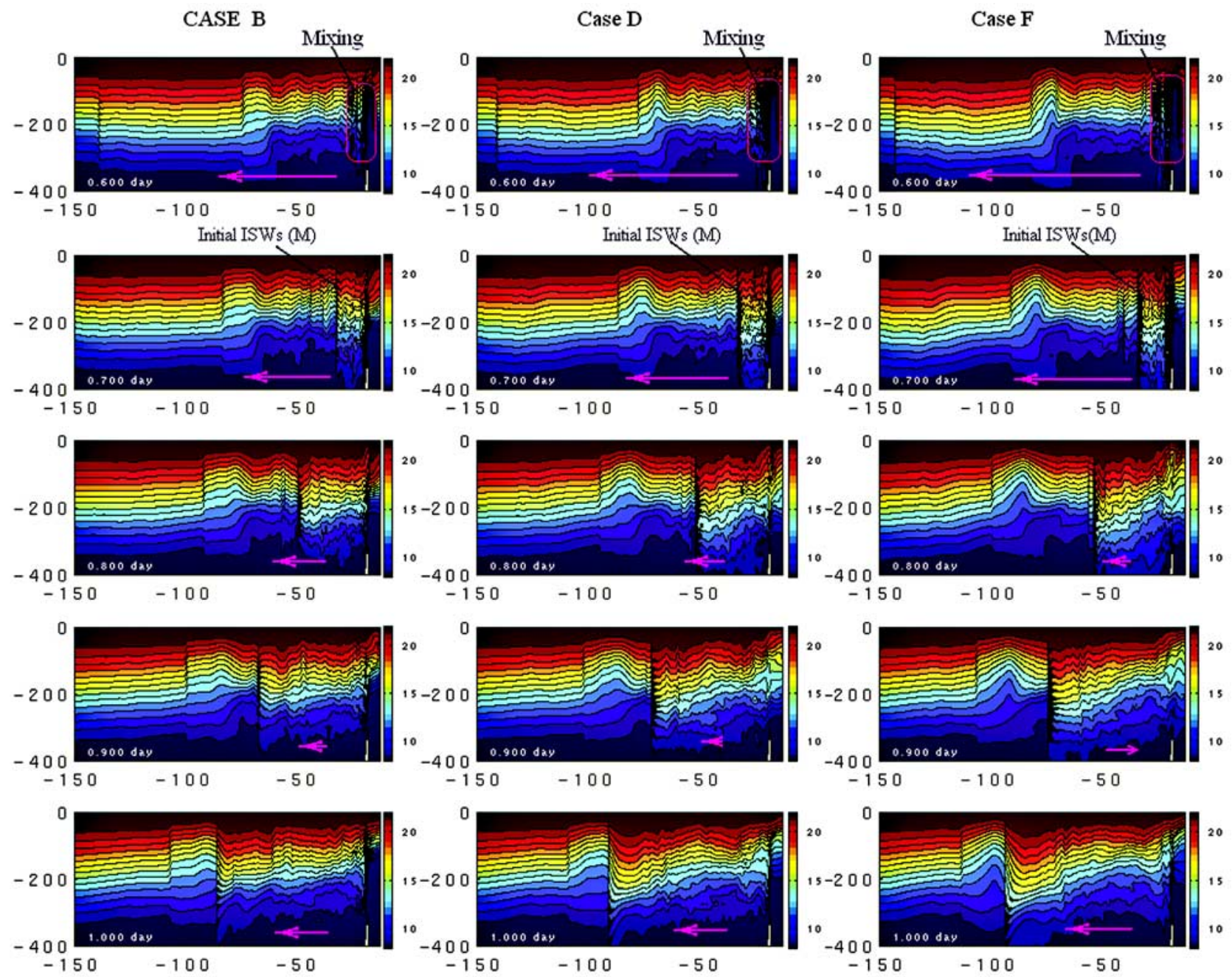

Figure 12. The temperature evolution showing the generation of ISWs within one tidal cycle (only a part of the simulation domain is shown) in different combined flow conditions with a strong K-branch entering LS: weak (left), medium (middle), and strong tide (right), respectively. The contour interval is $1^{\circ} \mathrm{C}$.

single ISW on SAR images (e.g., the ones in Figure 3). As the depression moves further westward, the single depression evolves into a group of ISWs (1.0 day). If a much stronger tide is imposed, the depression may quickly break down due to an extremely steep front, and generate intense internal mixing, which can bring about groups of ISWs as shown in Figure 12.

[25] In order to further explore the impacts of the Kuroshio intrusion on the generation of ISWs, a constant K-branch with different strengths is added at the eastern open boundary together with a variety of tidal strengths. To better illustrate these generation processes of ISWs, Figures 12 and 13 only show the upper $400 \mathrm{~m}$ region between the two seamounts. Comparing the simulations with (Figures 12 and 13) and without the K-branch (Figure 11), a vertical mixing region is generated in the west and upon the higher seamount in the cases with the K-branch. In Case B (Figure 12, left), a strong K-branch and a weak tide are imposed at the eastern boundary and this results in a vertical mixing region extending about10 $\mathrm{km}$ downstream of the higher seamount. The disturbance of the mixing water creates groups of ISWs at its western edge on 0.7 day (marked by ISWs (M)). The ISWs continue to grow by gaining more energy from the mixed water as the mixing area is extended. Finally, when the stratification re-establishes in the mixing area around 0.8 day, the generation of ISWs is completed. This location is defined as the actual source location of these ISWs. It is about $30 \mathrm{~km}$ downstream of the higher seamount.

[26] In Case D (Figure 12, middle), a strong K-branch, with the same strength as that in Case B, and a medium tide, with a tidal current as strong as the K-branch, are imposed. The combined flow now creates an area with strong vertical mixing region that extends to $10 \mathrm{~km}$ away downstream of the higher seamount on 0.6 day. Strong ISWs are initially generated by the disturbance of the mixing water at its western edge on 0.7 day (marked by ISWs (M)) and they continue to grow by gaining more energy from the mixed water as the mixing area is extended. The generation process of ISWs is completed when the stratification re- 


\section{Case $B^{\prime}$}
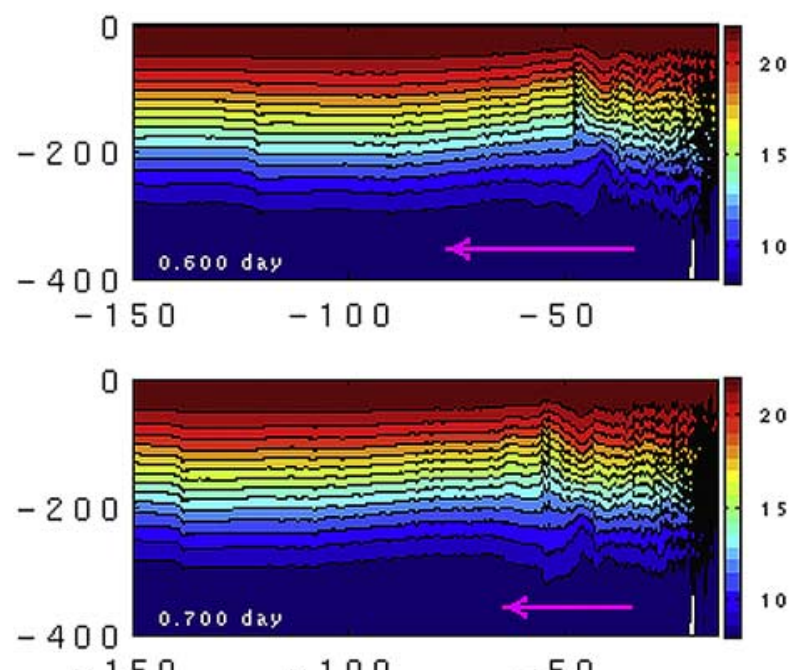

$$
\begin{array}{lll}
-150 & -100 & -50
\end{array}
$$

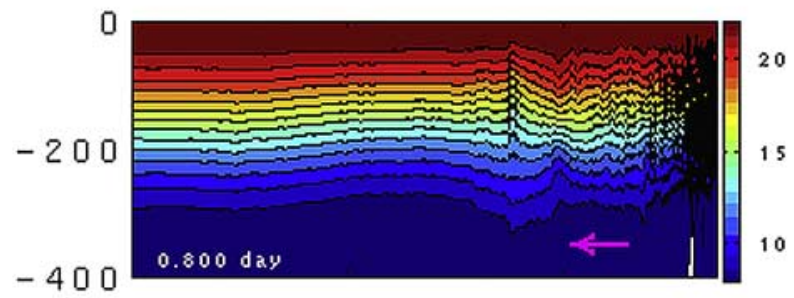

$$
-150 \quad-100 \quad-50
$$
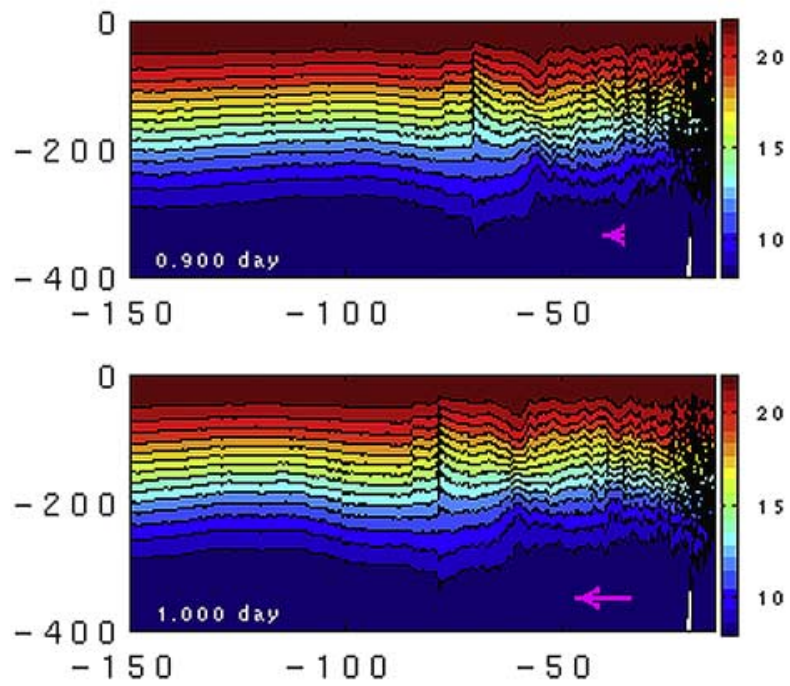

\section{Case $\mathrm{D}^{\prime}$}
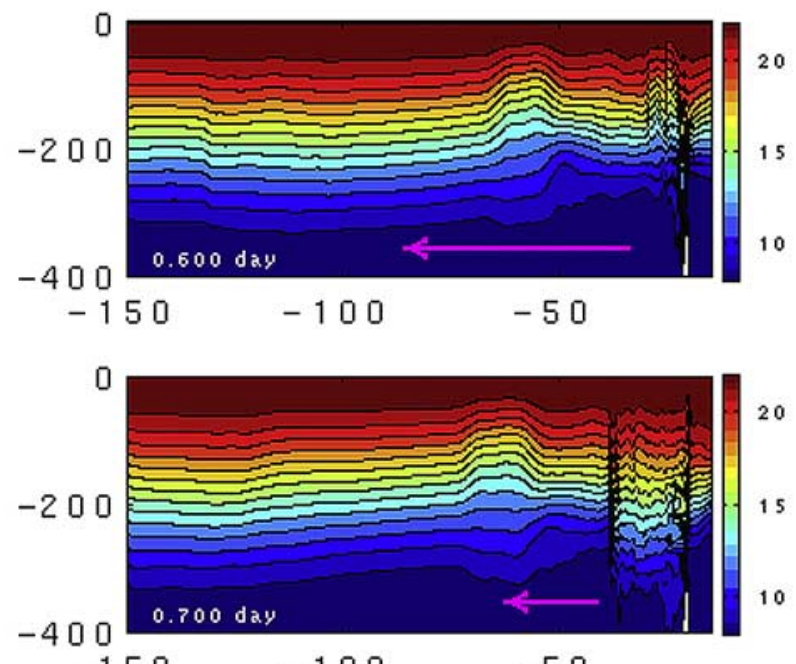

$-150$

$-100$

$-50$

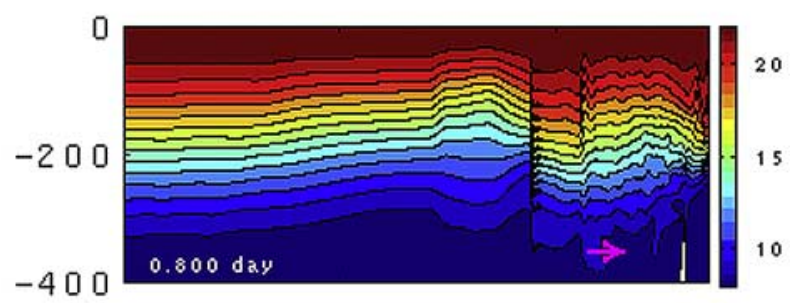

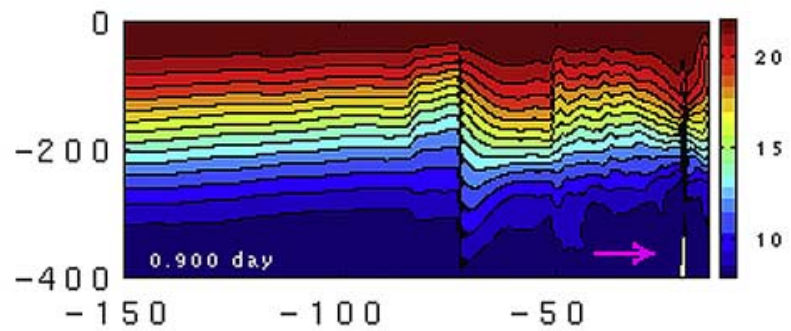

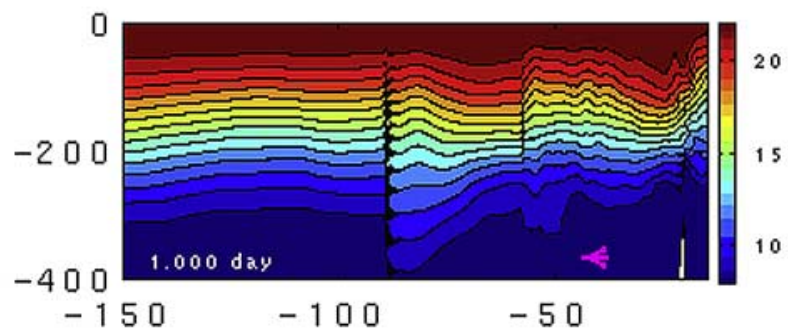

Figure 13. The temperature evolution showing the generation of ISWs within one tidal cycle (only a part of the simulation domain is shown) in different combined flow conditions. (left) A weak tide combined with a medium K-branch. (right) A medium tide combined with a weak K-branch. The contour interval is $1^{\circ} \mathrm{C}$.

establishes in the mixing area around 0.8 day. This location is defined as the actual source location of these ISWs. It is between 30 40 km downstream of the higher seamount.

[27] In Case $\mathrm{F}$ (Figure 12, right), the generation process of ISWs is similar to that in Cases B and D, but imposed on the east boundary are a strong K-branch, with the same strength as that in Case B, and a strong tide, with a tidal current stronger than the K-branch. The combined flow (the strongest flow condition among all simulations) creates an area with the most intense vertical mixing in Cases B, D, and $\mathrm{F}$. The disturbance of the mixing water creates strongest ISWs initially at the west edge of the area. Similar to those in Cases B and D, the ISWs continue to grow until stratification re-establishes in the mixing area after 0.8 day. This location, approximately $40 \mathrm{~km}$ from the top of the 
higher seamount, is defined as the actual source location for these ISWs as well.

[28] Figure 13 shows the generation of ISWs for Case B' (a weak tide is combined with a medium K-branch) and Case $\mathrm{D}^{\prime}$ (a medium tide is combined with a weak $\mathrm{K}$ branch), respectively. Similar to Case B, the combined flow in Case $B^{\prime}$ creates an area of vertical mixing, west and upon of the higher seamount, but the disturbance of the mixed water is not strong enough to generate any ISWs. While in Case $\mathrm{D}^{\prime}$, the combined current is not strong enough to bring about any mixing so that the generation process of ISWs is much closer to that in Cases $\mathrm{C}$ and $\mathrm{E}$, in which no K-branch was added (Figure 11, middle and right). In other words, ISWs are still generated by the evolution of a depression, instead of the disturbance of mixing water.

[29] In summary, these simulations clarify that the ISWs are most likely generated in the area between the two seamounts. This suggests that the lower seamount seems not very important for the generation of the ISWs and has minor effects on the propagation of ISWs toward the SCS. Another recent study on the modification of the whole west ridge of LS on ISWs [Chao et al., 2007] also supports the existence of such effects. The west ridge in the middle of LS, represented by the lower seamount in our simulations (Figure 10a), hinders but not annihilates the westward transmission of internal waves. A further comparison (not shown herein) of simulations with and without the lower seamount shows that the lower seamount is insensitive to the generation of ISWs in LS.

\section{Discussions}

\subsection{Impacts of Tidal Currents on the Generation of} ISWs in LS

[30] Figure 11 illustrates the generation processes of ISWs under different tidal current strengths. In Case A, the maximum tidal current $V_{m}$ on the top of the higher seamount is weak; hence, the interaction between the tidal current and the seamount does not cause ISW generation (Figure 11, left). In Case C, the maximum tidal current $V_{m}$ is already strong enough to bring about group of ISWs. While in Case E, the maximum tidal current $V_{m}$ becomes much stronger and it results stronger group of ISWs and also a single ISW (Figure 11, right). These processes suggest that ISWs can be generated only when the tidal current is strong enough and that the generation of stronger ISWs requires stronger tidal currents.

\subsection{Impacts of the K-Branch Current on the Generation of ISWs in LS}

[31] The impacts of the K-branch current on the generation of ISWs in LS are investigated by superimposing a constant current with different speeds to the weak, medium, and strong tides, respectively. The adding of the K-branch (always flow westward) to periodic tidal currents results in combined currents which not only are stronger than the original tidal currents, but also last longer when flowing westward. These combined currents make the generation of ISWs (Figures 12 and 13) different from that with only tidal currents (Figure 11) in most cases.

[32] In Case $\mathrm{B}^{\prime}$, the combined currents bring about no obvious ISWs although its combined current has a greater maximum velocity $\left(V_{m}=183 \mathrm{~cm} / \mathrm{s}\right)$ than the tidal current in Case C, where $V_{m}=146 \mathrm{~cm} / \mathrm{s}$ and ISWs are generated. When the combined currents become much stronger like in cases $\mathrm{D}\left(V_{m}=290 \mathrm{~cm} / \mathrm{s}\right)$ and $\mathrm{F}\left(V_{m}=326 \mathrm{~cm} / \mathrm{s}\right)$, they do bring about groups of ISWs (Figure 12, middle and right) and these ISWs are generated by the disturbance of internal mixing, instead of by the evolution of a depression like in Cases $\mathrm{C}$ and $\mathrm{E}$, where the depression was generated by tidal currents. The simulations in Figures 12 and 13 clearly show that the adding of the K-branch to tidal currents increases the frequency of inducing internal vertical mixing and initiating groups of ISWs by the disturbance of the internal vertical mixing, although the disturbance of the internal vertical mixing can initiate groups of ISWs only when the combined currents are strong enough.

[33] Since the K-branch can be remotely affected by many factors, its occurrence time, strength, and direction may vary rapidly. The strong $\mathrm{K}$-branch or its associated current also complicates the occurrence of groups of ISWs in LS. This might explain the irregularity of the occurrence of ISWs on each lunar day (Figures 6 and 7).

\subsection{The Generation Mechanisms of ISW in LS}

[34] The generation of ISWs resulting from a tidal current flowing over a three-dimensional ridge has been studied by Maxworthy [1979] using laboratory experiments. He showed the dependence of the generation of ISWs on tidal current strength (velocity). When the tidal current is weak, no ISWs can be generated. When the current is strong, a downstream depression is formed at first and then evolves into a sequence of ISWs. However, the depression can also break and cause internal mixing under strong current conditions. In this situation, ISWs can possibly be created by two mechanisms: the collapse of the internal mixed region and the nonlinear evolution of lee waves. The notable difference between them is that the ISWs generated by the evolution of lee waves only propagate in the upstream direction, while those created by the collapse of the mixed region can propagate in either direction.

[35] The situation in LS is more complicated than that in Maxworthy [1979]. First, the topography on the east and west sides of the Batan and Babuyan Islands is not symmetric (Figure 1). Second, a strong south-to-north current, the Kuroshio, occurs only on the east side of the islands. Third, the combination of the K-branch with tidal currents results in a current that is much stronger on westward direction than on eastward one so that the flow condition is quiet different on both sides of the islands.

[36] Our simulations clearly illustrate the generation of ISWs west of Batan and Babuyan Islands. Figure 11 shows that the ISWs can be generated in the form of a single ISW or groups of ISWs. The depressions that can bring about ISWs are actually caused by strong tidal currents (Figure 11, Cases $\mathrm{C}$ and E). Figure 12 shows the groups of ISWs can also be generated by the disturbance of internal vertical mixing due to the combined currents; while the simulations in Figure 13 show ISWs are not necessary generated by internal mixing if the combined currents are not strong enough.

[37] We further explain the two generation mechanisms of ISWs in LS using a simplified topography, which represents 


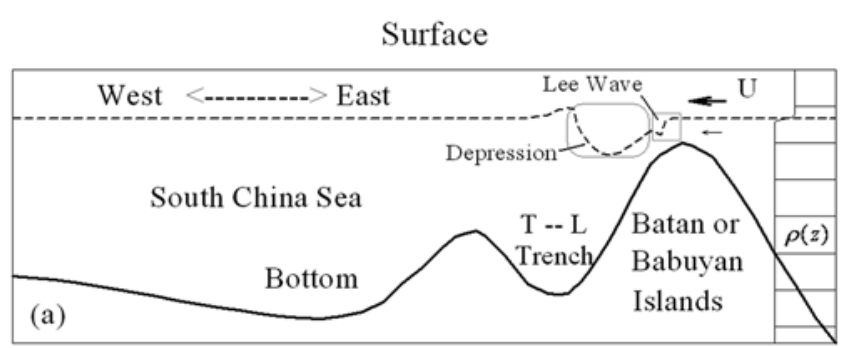

Surface

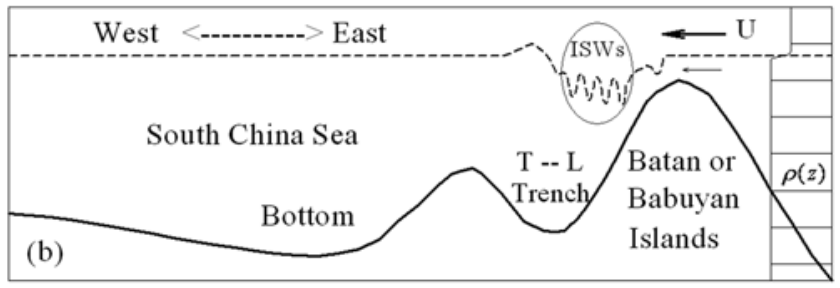

Surface

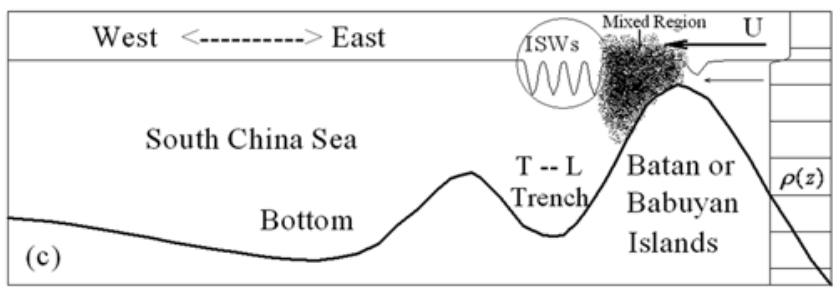

Surface

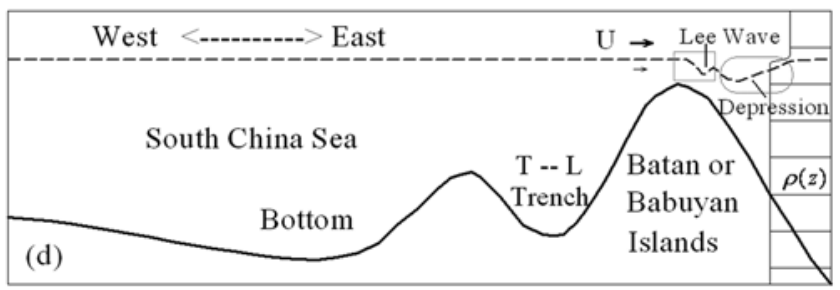

Figure 14. The generation models of ISWs resulting from a combined flow (tidal currents plus the K-branch) over the islands in LS. (a) The lee wave and the depression are formed by a weak westward flow U. (b) ISWs are generated from the depression front under a strong flow condition. (c) ISWs are generated from the disturbance of internal mixed waters under a stronger flow condition. (d) The lee wave and the depression are formed by a weak eastward flow U.

the east-west cross section of LS (Figure 14). The higher seamount represents the Batan and Babuyan Islands or the shallow ridge along $121.5^{\circ} \mathrm{E}$. The shallowest location of the ridge or the channels is supposed to be on the top of the seamount where tidal currents reach their maximum velocities.

[38] Let us focus on the west side of the Batan and Babuyan Islands first. When the combined current passes through the islands westward, mechanical stirring is produced by the interaction of the current with local topography, especially in the shallow and narrow channels among the islands (Figure 1) where the current reaches its maxi- mum velocity. If the maximum velocity of the combined current is low, the interaction can only create a weak downstream depression and some lee waves on the west side of the islands (Figure 14a). Before the westward combined current slackens, the lee waves will remain there while the depression continues to evolve westward. No ISWs will be generated from the evolution of the weak depression (like Case A in Figure 11). However, when the maximum velocity is sufficiently high, the interaction will result in steep depressions. Further evolution of the steep depressions will finally give rise to a single or groups of ISWs (Figure 14b, like Cases $\mathrm{C}$ and $\mathrm{E}$ in Figure 11). This kind of generation process of ISWs, evolved from a depression, has been studied numerically [e.g., Liu et al., 1998, 2004] and verified with remotely sensed images [Zhao et al., 2004]. If the maximum velocity is high enough, the depression will become extremely steep so that the depression (rather than evolving into ISWs) will quickly break down and bring about intense internal mixing. The disturbance of the mixed waters on its surrounding stratified waters will directly create groups of ISWs (Figure 14c, like the cases in Figure 12).

[39] As the westward combined currents slacken, some lee waves (Figures 14a and 14b) will propagate upstream (eastward). Similarly, some ISWs originating from the mixed waters (Figure 14c) will also propagate eastward. The eastward lee waves may evolve into eastward ISWs. Since we are mainly interested in ISWs propagating toward the SCS, these eastward propagating ISWs are beyond the scope of this study.

[40] On the east side of the Batan and Babuyan Islands, we believe the disturbance of the strong south-to-north Kuroshio and the attenuation of eastward tidal currents by the K-branch reduce the possibility of generating lee waves and internal intense mixing (Figure 14d). Therefore the westward propagating ISWs are less likely generated on this side. The remotely sensed images have not shown any tracks of these ISWs in the area over the islands till now. Furthermore, in order to reach the SCS, the westward propagating ISWs need a longer and harder journey to pass than those generated on the west side. Therefore these ISWs should be infrequently seen in SCS and will not be further stressed here.

\subsection{The Strength of Tidal Currents for the Generation of ISWs}

[41] The generation of ISWs in LS closely relates to the strength of tidal currents and the K-branch in our simulations. As shown by Maxworthy [1979], the strength of tidal currents was described by Froude number (Fr). When the Fr exceeds its supercritical $\mathrm{Fr}_{\mathrm{m}}$ (beyond which mixing occurs), ISWs can be created by two mechanisms; when Fr is below its subcritical $\mathrm{Fr}_{\mathrm{c}}$, no ISWs and no depression are produced; when $\mathrm{Fr}$ is between $\mathrm{Fr}_{\mathrm{m}}$ and $\mathrm{Fr}_{\mathrm{c}}$, ISWs can only be generated by the evolution of depression waves. However, how to determine these critical values in real ocean conditions is still unclear. Several recent studies [e.g., Laurent and Garrett, 2002; Legg, 2004] on internal tides or related phenomena suggested a single parameter $\mathrm{kU} / \omega$ to relate tidal excursion amplitude $U / \omega$ to the horizontal scale of the bathymetry $\mathrm{k}-1$, where $U$ is tidal current speed, $\omega$ is frequency, and $\mathrm{k}$ is the wave number for the bathymetry. 
When this parameter is less than unity, internal waves are likely generated at the fundamental tidal frequency; when it exceeds unity, a lee-wave mechanism involving higher harmonics in frequency is pertinent [Laurent and Garrett, 2002]. Unfortunately, this parameter cannot be applied in LS since our simulations show that the ISWs observed in LS are more like generated by other mechanisms instead of lee-wave mechanism.

[42] The critical values of the Froude number are not further investigated in this study since they are not simply determined by the currents only, but many other conditions like stratification and local topography etc. Our analysis in section 2 reveals the tidal currents in spring tides are strong enough to bring about ISWs in LS.

\section{Conclusions}

[43] Two types of ISWs are observed in LS: a single ISW and groups of ISWs; the ISWs occur more frequently during spring tides. Both the analysis of the remotely sensed images and the numerical simulations show that the strength of tidal currents and the K-branch has significant impacts on the generation of ISWs in LS: the stronger the currents, the more likely ISWs are generated, and the real tidal current or its combination with a K-branch in spring tides can sufficiently lead to the generation of ISWs in LS.

[44] The numerical simulations are used to verify two possible generation mechanisms of ISWs in LS: the evolution of a depression and the disturbance of internal vertical mixing to the surrounding water. The former mechanism can create (1) single ISW and (2) groups of ISWs while the later can only produce groups of ISWs. The later mechanism requires stronger currents for the generation of ISWs.

[45] Finally, two possible ISW sources are also identified in LS based on the analysis of the remotely sensed images and the numerical simulations, west of the Batan Islands and west of the Babuyan Islands. The distance between the observed ISWs and the islands suggests that ISWs may not be generated in or near the islands.

[46] Acknowledgments. The authors wish to thank Doug Salmon for the language improvement of this manuscript; Dong-Shan Ko, Naval Research Laboratory, for supplying us Figures 8 and 9; Werner R. Alpers, Guohong Fang, and Richard Field for reading the manuscript and for their constructive comments. We are grateful to the National Center for Highperformance Computing, Taiwan, for computer time and facilities. This research was supported partially by the Office of Naval Research (ONR) through grant N00014-03-0337, by the National Aeronautics and Space Administration (NASA) through grant NAG5-11773, and by the National Oceanic and Atmospheric Administration (NOAA) through grant NA17EC2449. Some support also comes from the Chinese National Basic Research Program 973: 2007CB416605. The second author acknowledged the support from National Science Council (grant 962628M002010), Taiwan.

\section{References}

Bole, J. B., C. C. Ebbesmeyer, and R. D. Romea (1994), Soliton current in the South China Sea: Measurements and theoretical modeling, paper presented at 26th Annual OTC in Houston, Tex., 2-5 May.

Centurioni, L. R., P. P. Niiler, and D. K. Lee (2004), Observations of inflow of Philippine Sea surface water into the South China Sea through the Luzon Strait, J. Phys. Oceanogr., 34, 113-121.

Chao, S. Y., D. S. Ko, R. C. Lien, and P. T. Shaw (2007), Assessing the west ridge of Luzon Strait as an internal wave mediator, J. Oceanogr., 63, 897-911.
Chu, P. C., and R. F. Li (2000), South China Sea isopycnal-surface circulation, J. Phys. Oceanogr., 30(9), 2419-2438.

Dietrich, D. E. (1997), Application of a modified "a" grid ocean model having reduced numerical dispersion to the Gulf of Mexico circulation, Dyn. Atmos. Oceans, 27, 201-217.

$\mathrm{Du}, \mathrm{T}$. (2000), The source of the internal waves in the northern part of the South China Sea (in Chinese), Front Line Earth Sci., 7(Suppl.), 188.

Duda, T. F., J. F. Lynch, and J. D. Irish (2004), Internal tide and nonlinear internal wave behavior at the continental slope in the northern South China Sea, IEEE J. Oceanic Eng., 29, 1-27.

Ebbesmeyer, C. C., C. A. Coomes, and R. C. Hamilton (1991), New observation on internal wave (soliton) in the South China Sea using acoustic Doppler current profiler, in Marine Technology Society Proceedings, pp. 165-175, Marine Technology Society, New Orleans.

Fang, G. H., Y-K. Kwok, K. Yu, and Y. Zhu (1999), Numerical simulation of principal tidal constituents in the South China Sea, Gulf of Tonkin and Gulf of Thailand, Cont. Shelf Res., 19, 845-869.

Fang, X. H., and T. Du (2005), Fundamentals of Oceanic Internal Waves and Internal Waves in the China Sea (in Chinese), China Ocean Univ. Press, QingDao, China.

Hsu, M. K., A. K. Liu, and C. Liu (2000), A study of internal waves in the China Seas and Yellow Sea using SAR, Cont. Shelf Res., 20, 389-410.

Laurent, L. S., and C. Garrett (2002), The role of internal tides in mixing the deep ocean, J. Phys. Oceanogr., 32, 2882-2899.

Legg, S. (2004), Internal tides generated on a corrugated continental slope. part I: Cross-slope barotropic forcing, J. Phys. Oceanogr., 34, 156-173.

$\mathrm{Li}, \mathrm{F} ., \mathrm{L}$. Li, X. Wang, and C. Liu (2002), Water masses in the South China Sea in summer and winter of 1998 and water exchange between the Pacific and the South China Sea, J. Ocean Univ. Qingdao, 32(3), 329336.

Lien, R.-C., T. Y. Tang, M. H. Chang, and E. A. D'Asaro (2005), Energy of nonlinear internal waves in the South China Sea, Geophys. Res. Lett., 32, L05615, doi:10.1029/2004GL022012.

Liu, A. K., S. R. Ramp, and Y. Zhao (2004), A case study of internal solitary wave propagation during ASIAEX 2001, IEEE J. Oceanic Eng., 29, 1144-1156.

Liu, A. K., S. Y. Chang, M.-K. Hsu, and N. K. Liang (1998), Evolution of nonlinear internal waves in East and South China Seas, J. Geophys. Res., 103, 7995-8008.

Liu, Q., H. Yang, and W. Li (2000), Velocity and transport of the zonal current in the Luzon Strait, Acta Oceanol. Sin., 22(2), 1-8.

Maxworthy, T. (1979), A note on the internal solitary waves produced by tidal flow over a three-dimension ridge, J. Geophys. Res., 84, 338-346.

Niwa, Y., and T. Hibiya (2004), Three-dimensional numerical simulation of $\mathrm{M}_{2}$ internal tides in the East China Sea, J. Geophys. Res., 109, C04027, doi:10.1029/2003JC001923

Orr, M. H., and P. C. Mignerey (2003), Nonlinear internal waves in the South China Sea: Observation on the conversion of depression internal waves to elevation internal waves, J. Geophys. Res., 108(C3), 3064, doi:10.1029/2001JC001163

Pacanowski, R. C., and S. G. H. Philander (1981), Parameterization of vertical mixing in numerical models of tropical oceans, J. Phys. Oceanogr., 11, 1443-1451.

Ramp, S. R., T. Y. Tang, and T. F. Duda (2004), Internal solitons in the northeastern South China Sea. part I: Sources and deep water propagation, IEEE J. Oceanic Eng., 29, 1157-1181.

Tseng, Y. H., and D. E. Dietrich (2006), Entrainment and transport in the three-dimensional idealized gravity current simulation, J. Atmos. Ocean. Technol., 23, 1249-1269.

Tseng, Y. H., and J. H. Ferziger (2001), Effects of coastal geometry and the formation of cyclonic/anti-cyclonic eddies on turbulent mixing in upwelling simulation, J. Turbul., 2, 014.

Tseng, Y. H., J. H. Ferziger, and D. E. Dietrich (2005), Regional circulation of the Monterey Bay region: Hydrostatic versus nonhydrostatic modeling, J. Geophys. Res., 110, C09015, doi:10.1029/2003JC002153.

Tseng, Y. H., and L. C. Breaker (2007), Nonhydrostatic simulations of the regional circulation in the Monterey Bay area, J. Geophys. Res., 112, C12017, doi:10.1029/2007JC004093

Venayagamoorthy, S. L., and O. B. Fringer (2007), On the formation and propagation of nonlinear internal boluses across a shelf break, J. Fluid Mech., 577, 137-139.

Yuan, Y., Q. Zheng, D. Dai, X. Hu, F. Qiao, and J. Meng (2006), Mechanism of internal waves in the Luzon Strait, J. Geophys. Res., 111, C11S17, doi:10.1029/2005JC003198.

Zhao, Z., and M. H. Alford (2006), Source and propagation of internal solitary waves in the northeastern South China Sea, J. Geophys. Res., 111, C11012, doi:10.1029/2006JC003644 
Zhao, Z., V. Klemas, Q. Zheng, and X.-H. Yan (2004), Remote sensing evidence for baroclinic tide origin of internal solitary waves in the northeastern South China Sea, Geophys. Res. Lett., 31, L06302, doi:10.1029/ $2003 \mathrm{G} 1019077$.

T. Du, Physical Oceanography Laboratory, Ocean University of China, 5 Yushan Road, Qingdao 266003, China. (taodu@ouc.edu.cn)
Y.-H. Tseng, Department of Atmospheric Sciences, National Taiwan University, No. 1, Sec. 4, Roosevelt Road, Taipei 106, Taiwan. (yhtseng@ as.ntu.edu.tw)

X.-H. Yan, Center for Remote Sensing, Graduate College of Marine Studies, University of Delaware, 209 Robinson Hall, Newark, DE 19716, USA. (xiaohai@udel.edu) 